\title{
Automatic Single-Organ Segmentation in Computed Tomography Images
}

\author{
Ruchaneewan Susomboon, Daniela Raicu, Jacob Furst \\ Intelligent Multimedia Processing Laboratory \\ DePaul University, Chicago \\ \{rsusombo,draicu,jfurst\}@cti.depaul.edu
}

\author{
David Channin \\ Department of Radiology \\ Northwestern University, Chicago \\ dsc@radiology.northwestern.edu
}

\begin{abstract}
In this paper, we propose a hybrid approach for automatic single-organ segmentation in Computed Tomography (CT) data. The approach consists of three stages: first, a probability image of the organ of interest is obtained by applying a binary classification model obtained using pixel-based texture features; second, an adaptive split-and-merge segmentation algorithm is applied on the organ probability image to remove the noise introduced by the misclassified pixels; and third, the segmented organ's boundaries from the previous stage are iteratively refined using a region growing algorithm. While we applied our approach for liver segmentation in 2-D CT images, a challenging and important task in many medical applications, the proposed approach can be applied for the segmentation of any other organ in CT images. Moreover, the proposed approach can be extended to perform automatic multiple organ segmentation and to build context-sensitive reporting tools for computer-aided diagnosis applications.
\end{abstract}

\section{Introduction}

Automatic analysis of images from various medical imaging modalities is necessary to increase the productivity of radiologists when interpreting and diagnosing hundreds of images every day. Image segmentation is an important first step in analyzing medical data and serves as a vital preliminary step in many imaging applications such as diagnosis, anatomical structure studies, treatment planning, and computer integrated surgery [1].

Most of the segmentation techniques approaches applied for segmentation in medical field are based on the gray-intensities. These approaches can be divided into thee main categories: the thresholding approach, the active contour mappings approach, and the model-based (deformable) approach. In the traditional thresholding approach, the segmentation is performed by grouping all pixels that pass the predefined intensity criteria into regions of interest [2]. The active contour (e.g. snake [3, 4]) is a boundary-based approach which deforms a manually chosen initial boundary towards the boundary of the object by minimizing the image energy function. While the thresholding approach has to be tuned in order to find the best values for the thresholds producing the right segmentation, the active contour approach has to deal with the manual selection of the initial points on the region's boundary. Furthermore, when the edge of an object is not sharply different from the background, the active contour approach by itself may not differentiate between the region of interest and background. To overcome this challenge, most recent work of active contour mappings combines the active contours with the level sets approaches [5]. While the thresholding and active contour approaches do not use any apriori information, the model-based approach uses a template to find the region of interest; the template can also be deformed using certain rules in order to deal with different scales and positions of the regions of interest [6, 7]. Since the model-based segmentation approach is heavily based on shape information, the approach may fail when detecting organs where some abnormalities (such tumors) are present.

To perform medical image segmentation, the graylevels alone may not sufficient as many soft tissues have overlapping gray-level ranges and, thus, the use of the properties of the corresponding anatomical structures is necessary to perform accurate medical image segmentation [8]. Since the shape of the same organ might be different across a sequence of 2-D axial slices or even more, across different patients, several texturebased segmentation approaches have been proposed as a way to quantify the homogeneity and consistency of soft tissues across multiple Computed Tomography slices.

There are a large number of texture-based segmentation algorithms in the literature; among the most commonly used segmentation algorithms based on the texture features are clustering techniques, region growing, and split-and-merge techniques. Segmentation using these traditional techniques requires considerable amounts of expert interactive guidance or does not incorporate any spatial modeling which can result in poor segmentation results.

We propose a hybrid approach for automatic singleorgan segmentation in Computed Tomography (CT) data. The approach consists of three stages: first, a probability image of the organ of interest is obtained by applying a binary classification model obtained using 
pixel-based texture features (the organ of interest is one class and all the other pixels form the unknown class); second, since the classifier model does not incorporate any spatial information, an adaptive split-and-merge segmentation algorithm is applied on the organ probability image to overcome this drawback and, thus, remove the noise introduced by the misclassified pixels; and third, the segmented organ boundaries determined at the end of the previous stage are iteratively refined using a region growing algorithm.

Since the proposed process of texture-based image mining through the set of classification rules learned in the first stage is done at the pixel-level, the technique is independent from the anatomical structure variation from patient to patient. Furthermore, our image mining approach allows to segment organs with irregular shapes produced by the presence of certain tumors or abnormalities in the organ of interest. This is possible because the classification rules will assign a label to a pixel based on the rules learned on the normal tissues at the training stage; any pixel within the region of interest receiving a low probability label will become a candidate pixel for a possible abnormality in the region. The regions with these irregular shapes will be analyzed further by the radiologists for abnormality detection.

While we applied our approach for liver segmentation in 2-D CT images, a challenging and important task in many medical applications (every year approximately 1,000,000 liver cancer cases are reported [9]), such as in surgical planning for liver transplants and in detection of pathological states [10], the proposed approach can be applied for the segmentation of any other organ in CT images. Moreover, the proposed approach can be extended to perform automatic multiple organ segmentation by combining the output from each individual single-organ segmentation filtering and to perform volumetric segmentation as well. Furthermore, the automatic detection of organs of interest in CT data will have a signification impact on building context-sensitive reporting tools (for instance, apply a computer-aided diagnosis (CAD) tool for liver only if the region of interest is a liver).

The rest of this paper is organized as follows. Our methodology is discussed in Section 2, the preliminary experimental results are presented in Section 3, and the conclusions are discussed in Section 4.

\section{Methodology}

Our approach for single-organ segmentation in CT images consists of three stages as shown in Figure 1. First, a probability image of the organ of interest is obtained by applying a binary classification model obtained using pixel-based texture features; second, an adaptive split-and-merge segmentation algorithm is applied on the organ probability image to remove the noise introduced by the misclassified pixels; and third, the segmented organ boundaries determined at the end of the previous stage are iteratively refined using a region growing algorithm.

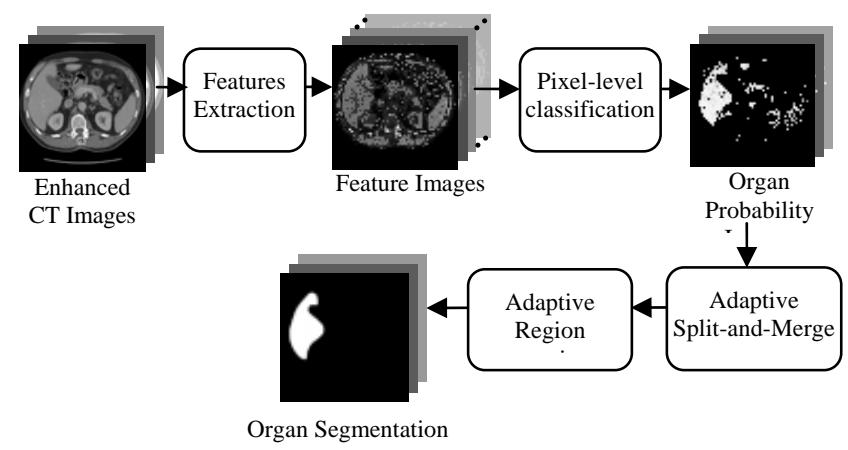

Figure 1. Diagram of the proposed approach

\subsection{Pixel-level texture extraction}

Pixel-level texture extraction is the process of analyzing and quantifying the texture patterns within a small neighborhood around the pixel of interest. There are several texture models, including structural, transform methods, and statistical models [11]; among all of these models, it has been shown that the secondorder statistical model (the co-occurrence matrix) produces better classification accuracy over the transform-based approach and structural method [12, 13]. The common technique used to extract texture information from the co-occurrence matrix model is based on the Haralick texture descriptors [14]. Figure 2 shows the steps performed in our texture feature extraction process.

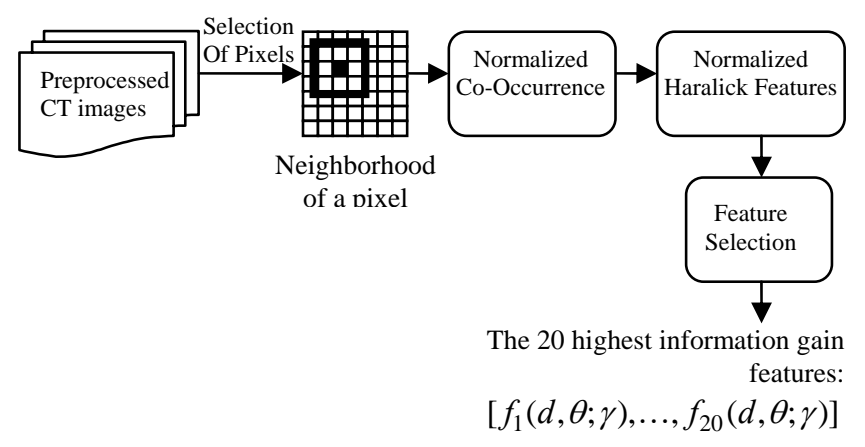

Fiaure 2. Pixel-level Texture Extraction Diaaram

For each pixel of interest a small neighborhood of size $\mathrm{N}$ by $\mathrm{N}$ is formed and the normalized co-occurrence matrix, $P_{i j}(d, \theta)$, of displacement vector $d$ and angle $\theta$, where $i$ represent the gray-level at vertical direction (row) and another $j$ gray-level at horizontal direction (column). In order to capture all possible texture 
patterns, we calculate all possible displacements $(\mathrm{N}-1)$ for four directions $\left(0^{\circ}, 45^{\circ}, 90^{\circ}, 135^{\circ}\right)$ within the corresponding neighborhood.

Once the co-occurrence matrix, nine Haralick texture descriptors (entropy, energy, contrast, sum average, variance, correlation, maximum probability, inverse different moment, and cluster tendency) are calculated. In order to reduce the feature set and select the most important features, an information-gain criterion [15] is applied on the feature set. At the end of this stage, each pixel will be characterized by a p-dimensional feature vector, where indicates the number of most discriminative features, and represent the displacement and the angle, respectively, and indicates the corresponding Haralick feature. The feature vector is further normalized by a min-max normalization technique applied with respect to each individual feature.

\subsection{Pixel-based texture classification}

In our approach, a binary Classification and Regression Tree (C\&RT) model is applied on the pixellevel data generated at the previous step in order to generate decision rules for the classification of the organ of interest. The C\&RT model is chosen because it does not make any assumptions about the distribution of the data, its computational efficiency, easy interpretability of the output data in the form of decision rules, and good classification performance as shown in [12]. Once the optimal decision tree is built, each terminal node will produce a decision rule for the organ of interest and a probability will be associated with each rule in the form of IF-THEN PROBABILITY expression; the probability will be given by the ratio between the number of true organ pixels classified as the organ of interest and total number of pixels found at that terminal node. For instance, a rule as

$\operatorname{IF}\left[f_{1}(d, \theta ; \gamma)<0.34,0.65<f_{5}(d, \theta ; \gamma) \leq 0.8\right]$

THEN Liver with

PROBABILITY=.9,

denotes a rule obtained from a terminal node in which $90 \%$ of the pixels found at that node where indeed organ pixels.

At the end of the classification process, the decision rules will be applied to each pixel within the slice to be segmented; the output will be an organ probability image that will be used later in the adaptive split-andmerge segmentation stage; the probability image will also be used in the region growing stage in which pixels with lower intensities will be added as the algorithm advances from the center of the organ towards the boundary.

\subsection{Adaptive Split \& Merge segmentation}

Pixel-level classification is often an ultimate objective for image segmentation [16, 17]. However, classifiers generally do not perform any spatial modeling and this makes the segmentation process sensitive to the noise produced by the misclassified pixels.

To overcome this limitation, we propose an adaptation of the split-and-merge algorithm that will be applied on the organ probability image. The algorithm involves two main steps: 1) the selection of initial seed regions and 2) the selection of adjacent regions.

First, the image is split into smaller regions until all the regions meet a homogeneity criterion set by the threshold $\tau$ for splitting or the region size is smaller than a certain threshold. The homogeneity criterion is based on the average of the organ probabilities within the region considered for splitting; if the average probability is smaller than the threshold, the region is split further unless its size is smaller than a certain value. Second, the small split organ regions are grouped to form the organ of interest if they are adjacent to each other. In other words, the image is segmented into disjoint regions that correspond to the two classes, the organ of interest class and the unknown class (all the other tissues).

The threshold for splitting is a very important parameter. Its value will be set very high in order to find a reliable seed set (pixels close to the center of the organ region are expected to have high organ probabilities). Therefore, we allow the threshold to vary only at $90 \%$ and set the minimum region size of $16 \times 16$ in order to attain reliable.

Once the seed set is determined, the second step of selecting adjacent regions takes place; the process looks only at the regions adjacent to the seed set and splits these regions further if the two relaxed splitting criteria (decreased probability threshold and a new minimum sub-region size) are not satisfied. Again, the homogeneity threshold is relaxed down to $80 \%$ confidence since a lower level will introduce non-organ pixels within the segmented region. The cutoff value for the splitting threshold is chosen automatically based on the sensitivity of the classifier with respect to the organ of interest. This potentially early termination (because of generally high sensitivities) may cause the system not to include some of the organ boundary pixels and so a refinement stage is necessary as described in the following section. 


\subsection{Adaptive region growing}

To refine the boundary of the segmented organ, an adaptive region growing approach is deployed as a postprocessing step. First, the process attempts to find the edge-pixels from segmented regions. Around each edgepixel, a window of size $\mathrm{N}$ by $\mathrm{N}$ is formed and the average probability within that window is calculated. The edge-pixel will be merged with the segmented region, only it satisfies the region constraint. The threshold for refinement is more relaxed compared with those used in the process of splitting since, at the organ boundary, the average of the organ' probabilities tends to be lower. We allow the threshold to go down up to $60 \%$ (lower than this will allow results obtained by chance) and the process repeats until no new pixel is added in the result of the region segmentation.

\section{Preliminary results}

Our preliminary results are based on data extracted from normal CT studies from Northwestern Memorial Hospital (NMH) PACS. The data consists of multiple, serial, axial computed tomography images derived from helical, multi-detector CT abdominal and chest acquisitions using a HiSpeed CT/i scanner (GE Medical Systems, Milwaukee, WI). The images were transferred via Ethernet to a nearby computer workstation in DICOM format of size 512 by 512 and having 12-bit gray level resolution.

In order to obtain the prior knowledge for C\&RT, we randomly selected pixels from one CT slide in which the organ of interest was present such that $50 \%$ of total pixels are from the organ of interest class and another $50 \%$ of total selected pixels are from "unknown" class. The texture features for each pixel were calculated based on a 9 by 9 neighborhood determined experimentally to be the optimal one for producing the best pixel-level classification results for liver. In order to evaluate the model, the data was randomly divided into $66 \%$ for training and 34\% for testing. The optimal C\&RT was found at approximately $88 \%$ sensitivity and over $96 \%$ for the specificity on the testing set.

Figure 3 illustrates all stages of our proposed approach for segmenting the liver in a CT slice. The initial seed sets were obtained setting the threshold at 95\% (Fig. 3(b)) and then, the adaptive split-and-merge step was applied and repeated until the threshold was equal to $80 \%$ (Fig. 3(c)). After that, the region growing refined the boundary of the liver by varying the corresponding threshold down to 60\% (Fig. 3(d-e)).

While the classification rules were derived using pixels from a certain CT slice, we applied the rules to a sequence of consecutive slices in which the organ of interest was present. Fig. 4 shows the segmentation results; besides capturing very well the pixels belonging to liver and thus, delineating a good shape for the organ of interest, our approach is also able to differentiate among 'pure' liver pixels and pixels which belong to vessels or other structures present in the liver.

We also compared our approach with an intensitybased threshold approach. As shown in Figure 5, we noticed that it is hard to segment the liver from the other organs based on intensities only because of the overlapping gray levels among the soft tissues.

In contrast, our approach allows both mining the pixels representing liver and assigning high probabilities values to pixels close to the core of the organ and low values to the pixels approaching the boundaries. This type of probability assignment also mimics the segmentation produced by multiple radiologists: while radiologists will agree on the core of the organ, there will be a significant variation for the pixels close to the boundaries of the organ.

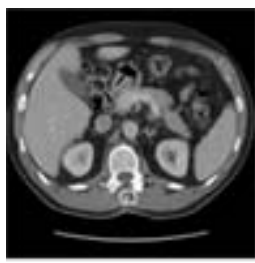

(a)

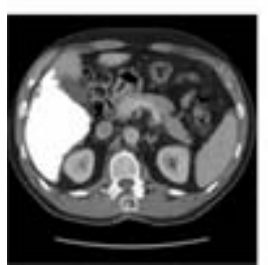

(d)

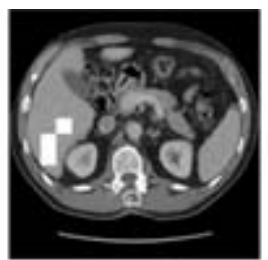

(b)

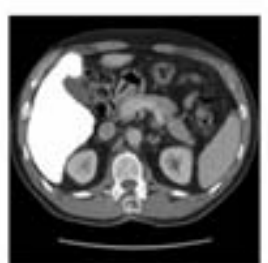

(e)

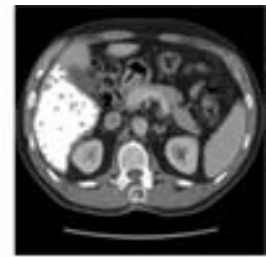

(c)

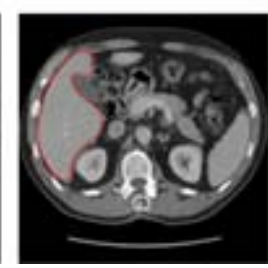

(f)
Figure 3. Example of the liver segmentation: (a) original image; (b) initial seed sets at $95 \%$ (c) split and merge for $80 \%$ threshold, respectively; $(d-e)$ images after region-growing at $70 \%$ and $60 \%$ thresholds, respectively; and (f) boundary of the segmented liver. 

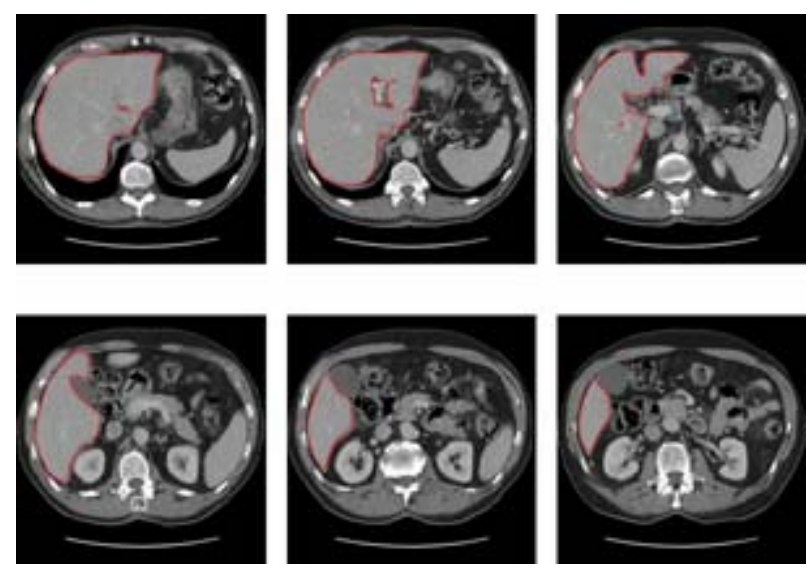

Figure 4. Examples of liver segmentation

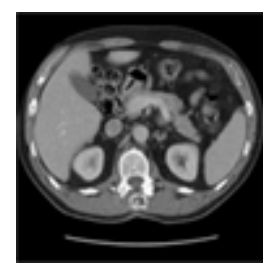

(a)

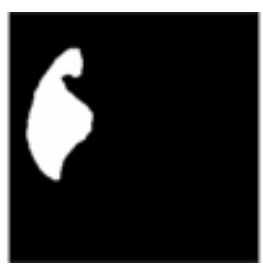

(b)

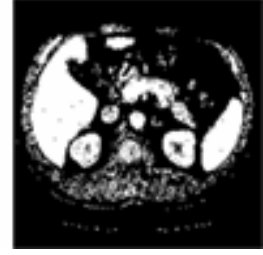

(c)
Figure 5. (a) Comparison of segmentation (a) original image; (b) our approach; (c) intensity-based thresholding

\section{Conclusion and future work}

Our preliminary results show that the liver can be correctly segmented within CT scans. While we applied our approach for liver segmentation in 2-D CT images, the proposed approach can be applied for the segmentation of any other organ in CT images since the presented approach did not use any liver specific information. Moreover, the proposed approach can be extended to perform automatic multiple organ segmentation and to perform volumetric segmentation as well. Furthermore, the automatic detection of organs of interest in CT data will have a signification impact on building context-sensitive reporting tools for computeraided diagnosis (CAD) applications.

\section{References}

[1] S.D. Olabarriaga, and A.W.M. Smeulders, "Interaction in the segmentation in medical images: A survey”, Medical Image Analysis, Vol. 5, No. 2, 2001, pp. 127-142

[2] P.K. Sahoo, S. Soltani, and A.K.C. Wong, "A Survey of Thresholding Techniques”, Computer Vision, Graphics and Image Processing, Vol. 41, No. 2, 1988, pp. 233-260
[3] M. Kass, A. Witkin, and D. Terzopoulos, "Snakes Active Contour Models”, International Journal of Computer Vision, Vol. 1, No. 4, 1987, pp. 321-331

[4] C. Xu and J.L. Prince, "Gradient Vector Flow: A New External Force for Snakes", IEEE Conf. on Computer Vision and Pattern Recognition, Los Alamitos: Comp. Soc. Press, June 1997, pp. 66-71

[5] P. Lin, C.X. Zheng, Y. Yang, and J.W. Gu, "Statistical Model Based on Level Set Method for Image Segmentation", The Fourth International Conference on Computer and Information Technology, 2004, pp. 143148

[6] D. Freedman, R.J. Radke, T. Zhang, Y. Jeong, D.M. Lovelock, G.T.Y. Chen, "Model-based segmentation of medical imagery by matching distributions”, IEEE Trans. Med. Imaging, Vol. 24, No. 3, 2005, pp. 281-292

[7] C. Xu, D.L. Pham, and J.L. Prince, "Medical Image Segmentation Using Deformable Models", SPIE Handbook on Medical Imaging, Vol. 3, Medical Image Analysis, edited by J.M. Fitzpatrick and M. Sonka, 2000

[8] R.N. Dave, and T. Fu, "Robust shape detection using Fuzzy clustering”, Practical application Fuzzy Sets Syst., Vol. 65, 1994, pp. 161-185

[9] R. Lu, P. Marziliano, and C. H. Thng, "Liver Tumor Volume Estimation by Semi-automatic Segmentation Method”, Proceedings of the 27th Annual International Conference of the IEEE Engineering in Medicine and Biology Society, 2005

[10] E. Sorantin, G. Werkgartner, R. Beichel, A. Bornik, B. Reitinger, M. Riccabona, R. Wegenkittl, and A. Fuhrmann, "The virtual liver surgery planning system", In European Congress of Radiology, The Matrix , 2004

[11] K.I. Chang, K.W. Bowyer, and M. Sivagurunath, "Evaluation of texture segmentation algorithms", IEEE Conf. on Computer Vision and Pattern Recognition, 1999, pp. 294-9

[12] M. Varma, and A. Zisserman, “Texture classification: are filter banks necessary?”, In Proc. IEEE Conference on Computer Vision and Pattern Recognition, Vol. 2, 2003, pp. 691-8

[13] J. Weszka, C. Deya, and A. Rosenfeld, “A Comparative Study of Texture Measures for Terrain Classification”, IEEE Trans. System, Man and Cybernetics, Vol. 6, 1976, pp. 269-285

[14] R.M. Haralick, K. Shanmugam, and I. Dinstein, “Textural Features for Image Classification”, IEEE Trans. on Systems, Man, and Cybernetics, Vol. Smc-3, no.6, 1973, pp. 610-621

[15] Sonka, M., Hlavac, V., Boyle, R., Image Processing, Analysis, and Machine Vision, Pacific Grove: Brooks/Cole Publishing Company, 1999

[16] D.L. Pham, C. Xu, and J.L. Prince, "Current methods in medical image segmentation”, Annual Review of Biomedical Engineering, Vol. 2, 2000, pp. 315-337

[17] D.S. Raicu, "Mining Knowledge in Computer Tomography Databases", Multimedia Data Mining and Knowledge Discovery, Springer Publishers. Valery A. Petrushin and Latifur Khan (Eds.), 2007. 